\title{
DESCRIPTION OF NEW INFLATABLE/RIGIDIZABLE HEXAPOD STRUCTURE TESTBED FOR SHAPE AND VIBRATION CONTROL
}

\author{
O. Adetona , L.H. Keel, Tennessee State University \\ L.G. Horta, Structural Dynamic Branch, NASA Langley Research Center \\ D.P. Cadogan, G.H. Sapna, S.E. Scarborough, ILC Dover, Inc.
}

\begin{abstract}
Larger and more powerful space based instruments are needed to meet increasingly sophisticated scientific demand. To support this need, concepts for telescopes with apertures of 100 meters are being investigated, but the required technologies are not in hand today. Due to the capacity limits of launch vehicles, the idea of deploying, erecting, or inflating large structures in space is being considered. Recently, rigidization concepts of large inflatable structures have demonstrated the capability of weight reductions of up to $50 \%$ from current concepts with packaging efficiencies near $80 \%$. One of the important aspects of inflatable structures is vibration mitigation and line-of-sight control. Such control tasks are possible only after actuators/sensors are properly integrated into a rigidizable concept. To study these issues, we have developed an inflatable/rigidizable hexapod structure testbed. The testbed integrates state of the art piezo-electric self-sensing actuators into an inflatable/rigidizable structure and a flat membrane reflector. Using this testbed, we plan to experimentally demonstrate achievable vibration and line-of-sight control. This paper contains a description of the testbed and an outline of the test plan.
\end{abstract}

\section{FLAT PRIMARY MIRRORS}

Space based conventional parabolic mirrors with apertures of about 100 meters are impractical using current technology. Fortunately such a large mirror can be approximated by smaller curved mirrors flying in formation. However, manufacturing each of these curved mirrors to the required precision, and subsequently controlling the surface of each segment to maintain the desired individual shapes is almost impossible.

An easier approach is to approximate each of these curved segments with a flat mirror. If these flat mirrors are circular with diameter $d$, they can be oriented to reflect signals from a distant source to a region of diameter $d$ around the focal point of the approximated mirror. Therefore, a concave secondary mirror with diameter $\mathrm{d}$ placed at this focal point can be used to focus the reflected signal onto a deformable tertiary mirror with diameter less than d. Errors in the wavefront of signals reflected from the secondary mirror can be negated by appropriately adjusting the flatness of the tertiary mirror. Note that these deviations can be measured using wavefront sensors and are partly due to the deviation of the primary mirror from a perfect parabolic surface.

Clearly membranes supported by inflatable structures are a good candidate for these flat mirrors. However standard testing, modeling and control techniques can not be used to achieve and maintain the tight membrane flatness requirements. Therefore static and dynamic tests must be performed on a membrane supported by inflatable structures to develop novel testing procedures and validate analytical models needed to develop new control systems. Details of a new testbed and an overview of planed tests are provided herein.

\section{DESCRIPTION OF THE TESTBED}

The structure is fabricated from inflatable, rigidizable thin-walled composite tubes. The use of inflatable structures allows the system to be packaged into a small volume for launch, yet deploy to hundreds of times that volume (depending on the structure) once in space. The testbed consists of a torus section, which suspends a flat membrane, and 6 struts, which mount on the torus and support a triangular shaped primary aperture.

The torus section is composed of twelve 0.181meter diameter graphite/epoxy composite beams, which are joined with rigid plastic joints. The composite is encapsulated by polyimide facesheets. The primary support struts are tapered from 0.0795-meters in diameter to 0.130-meters in diameter and are fabricated from the same graphite/epoxy composite material as the torus beams. Figures 1 through 4 show the assembled testbed.

The matrix resin used in all of the struts is a lightly cross-linked epoxy developed by ILC-Dover, Inc. When heated above its glass transition temperature (Tg), the modulus of the resin decreases allowing 
the entire composite strut to be flattened and rolled or $\mathrm{z}$-folded for packing.

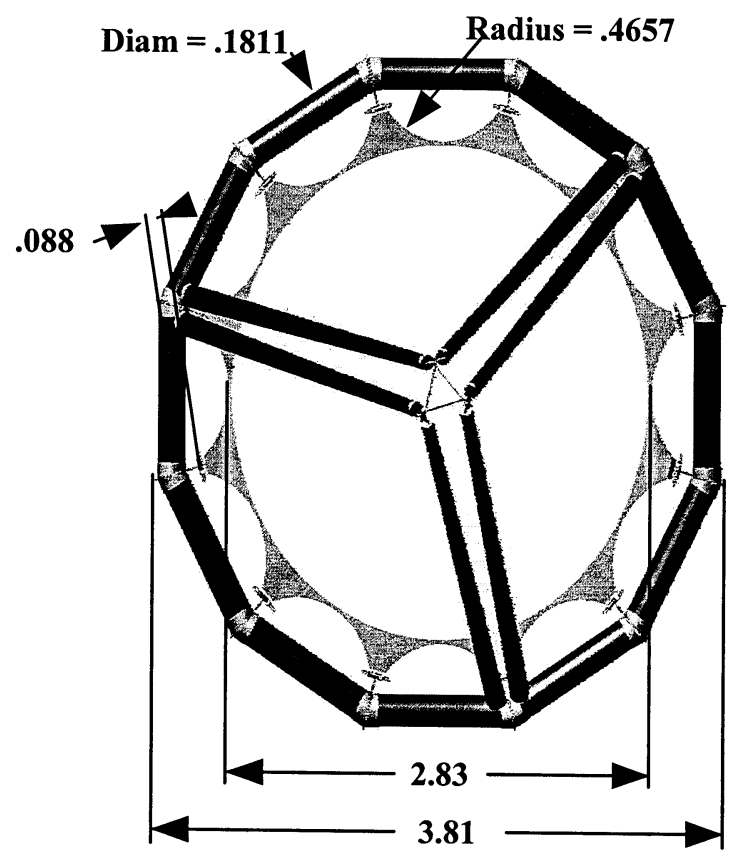

Figure 1. Torus and Membrane Dimensions (meters)

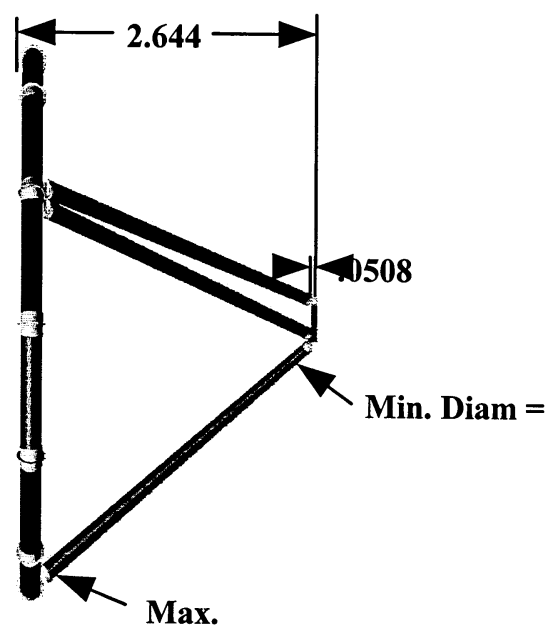

Figure 2. Dimensions of Tapered Primary Support

To deploy the structure, heat is applied to raise the structure's temperature above the resin's Tg. Next, inflation gas is introduced into the inflatable chambers of the structure. The structure takes shape as the gas fills the tubes. Once the structure is fully deployed, it cools to a temperature below the $\mathrm{Tg}$ of the resin, which causes the structure to become rigid again. Subsequently, the inflation gas is passively vented. This process eliminates the reliance on the inflation gas for structural support.

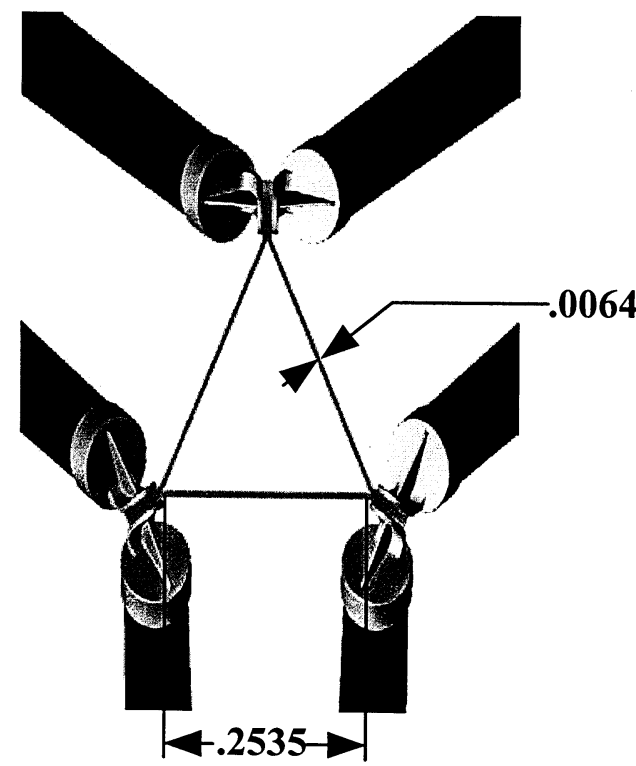

Figure 3. Primary Dimensions (meters)

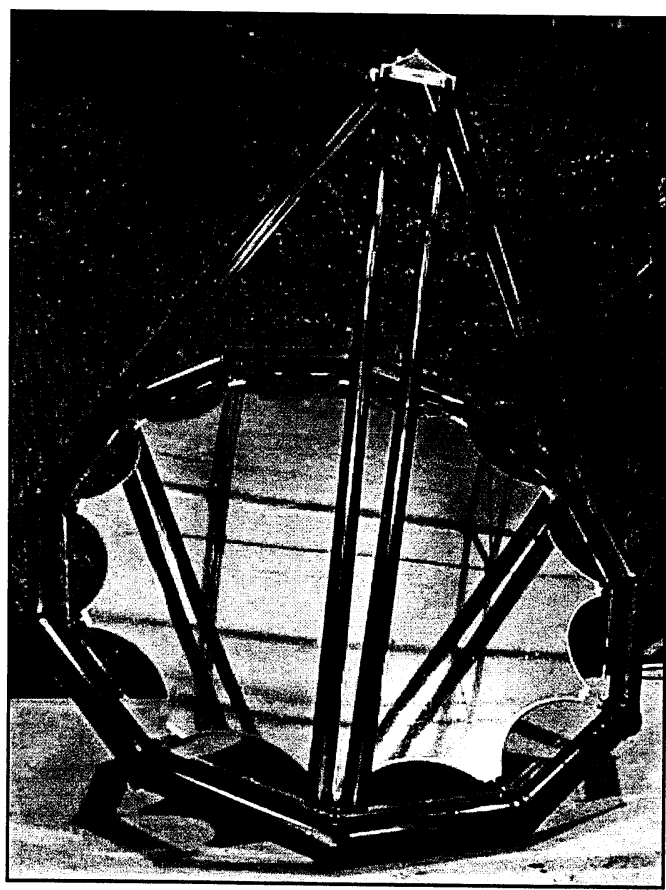

Figure 4. Assembled Hexapod 


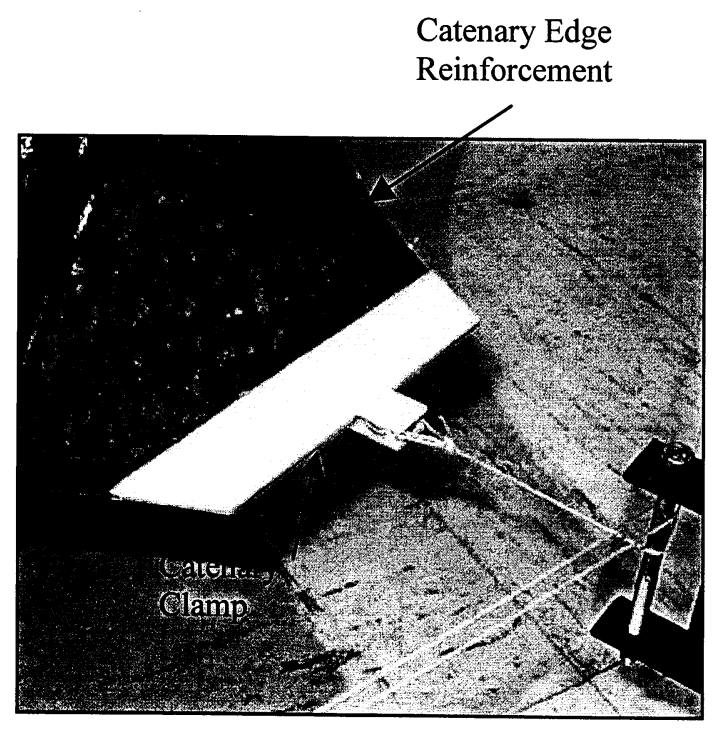

Figure 5. Catenary Tensioning System

For the testbed, the graphite composite tubes were joined with cast glass-filled urethane end caps. The endcaps provided the rigidity and geometry necessary to make the connections. The triangular shaped aperture at the top of the antenna (see Figure 4) was machined from 6061-T6 aluminum.

The dimensions of the testbed are provided in Figures 1-3.

\section{STRUCTURE ACTUATION DEVICES}

Deployable inflatable structures used for optical applications will require a method of damping vibrations from the structure. Embedding or bonding piezoelectric flexible patch actuators onto the structure allows for active control of the structural vibrations. LaRC micro-fiber composite MFC ${ }^{\mathrm{TM}}$ actuators, shown in Figure 6, will be bonded onto the wall surfaces of the composite struts, as shown in Figure 7.

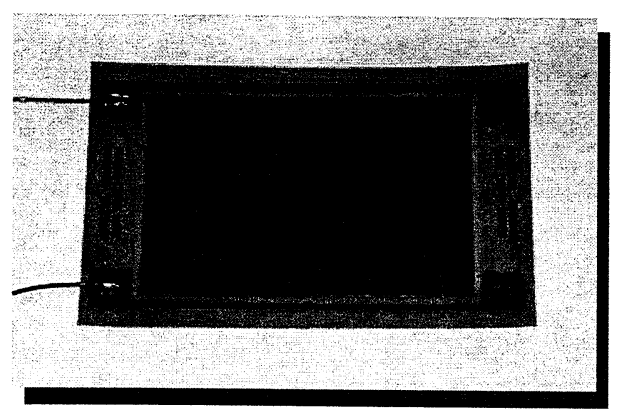

Figure 6. LaRC MFC Actuator

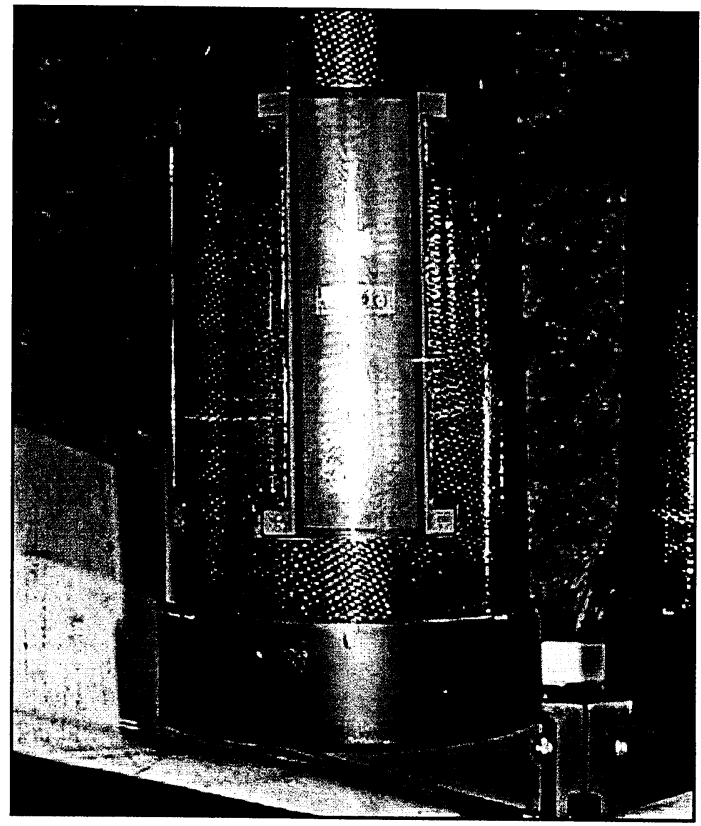

Figure 7. LaRC MFC Actuator Installed on Tapered Tube

The LaRC Macro-Fiber Composite (LaRC-MFC) is a low-cost, high performance, long life, and conformable piezoceramic actuator device. Each device is produced using low-cost sheets of prealigned piezoceramic fibers laminated between copper-polyamide electrode films. A scheme for the optimal location of these actuators will be developed early in the effort to determine the best method of controlling structural vibrations.

\section{MEMBRANE ACTUATION}

Static shape and vibration control of membranes are critical for applications where the membrane is used as a part of an optical system. Applications with less stringent surface quality requirements such as those sought for microwave communications require surface quality to be kept within 1-2 $\mathrm{mm}$ (Ref. 2). Actuation of membrane surfaces is an active area of research but our approach in this investigation is to use actuators at the membrane boundary for both in-plane and outof-plane actuation.

Figure 8 shows an expanded view of a section of the torus structure with a new membrane actuator mounted at the interface location between the membrane and the torus. These actuators are currently being designed to allow for actuation inplane using a stack of piezoelectric material in a 
cable pull arrangement. Out-of-plane motion is obtained by actuating piezo material, bonded onto the connecting flexure, to move the actuator housing. The end effect is similar to that observed in commercially available proof-mass actuators.

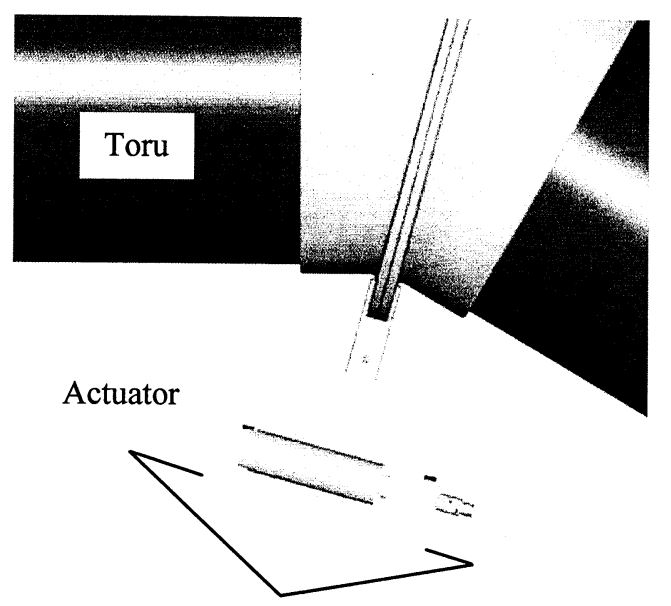

Membrane

Figure 8. Expanded View of Installed Membrane Actuator

\section{TEST PROCEDURE}

One of our goals is to develop test methods to generate data needed to validate analytical models. To accomplish this objective, structural components (i.e. the membrane, the toroid and individual struts) will be tested, followed by a test of the fully assembled structure. These static and dynamic tests must be performed using specially designed non-intrusive techniques. This is because traditional testing methods are generally not suitable to structures with low mass and low stiffness. To simulate a zero gravity environment, the tested structure/component will be supported in a manner that minimizes the effect of gravity on it. Accordingly the configuration shown in Figure 9 will be used to test the fully assembled structure because it allows the undisturbed membrane to lie in the vertical plane.

The dynamic characteristics (e.g transfer functions and vibration modes) of the structure will be studied by exciting the structure in three ways. For each source of excitation, an impulse, random and sine sweep signal will be imparted to the structure. The quality of data obtained will be used to determine the merits of each approach and the best approach (if any).

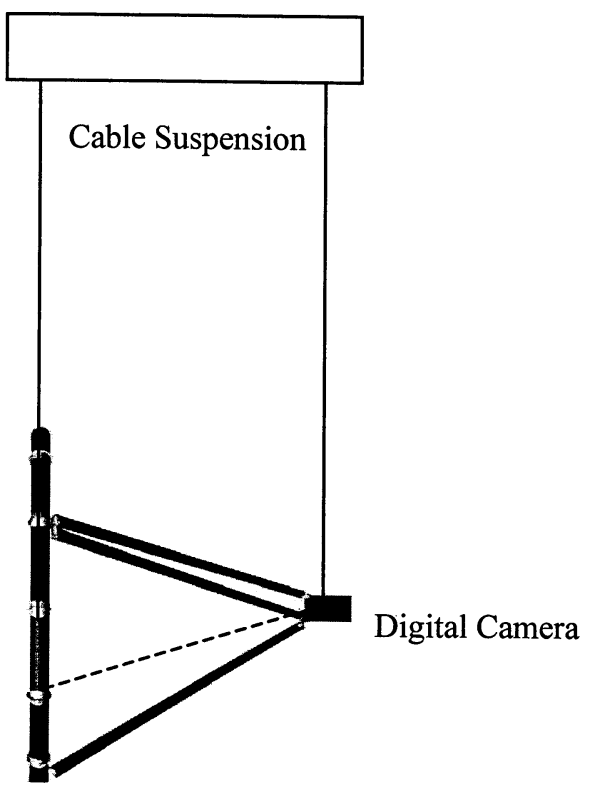

Figure 9. Vertically Suspended Configuration

The first method involves using an electrodynamic shaker to excite one of the interfaces between the toroid and a pair of struts. Secondly the membrane actuators will be used to excite the membrane. Hopefully the force from these actuators will also be enough to excite the entire structure. In the third approach, MFC actuators attached to the struts will be used to excite the struts and thereby excite the entire structure. Dynamic characterization using built-in MFC actuators is relatively new and is expected to yield interesting results.

Due to undesirable mass loading effects, an accelerometer should not be used on the membrane. Therefore, a scanning laser vibrometer will be used to measure the velocities of uniformly distributed points on the membrane surface. The velocities of unmeasured points/regions on the membrane can then be determined by interpolation using the velocities of neighboring points. This data will be used to provide a velocity profile and dynamic characterization of the membrane.

Unlike the membrane, the dynamic behavior of the toroid and struts will not be significantly affected by the presence of miniature accelerometers. Therefore, such accelerometers will be attached to strategic locations on these structures. Data from these accelerometers will be collected and 
recorded simultaneously. This data will be combined with the laser vibrometer data (for the membrane) and used for dynamic characterization of the entire structure.

Non-intrusive measurement of the static surface profile of the membrane surface is a big challenge. A promising technique for solving this problem was presented in Ref. 1. In that paper, digital cameras and photogrammetry (the science of measuring the dimensions of physical objects using pictures) were used to measure the membrane surfaces. Therefore, an initial evaluation of the static membrane shape will be conducted using the techniques described in Ref. 1. As an extension of the technique in Ref. 1, sets of pictures taken simultaneously by four digital cameras will be used to study the mode shapes of the structure after it has been excited.

\section{CONCLUDING REMARKS}

Satisfying the stringent requirements of some future space missions will require structures of extraordinary size. Membrane and Inflatable/rigidizable structures technology provides a relatively cheap and practical way to manufacture and deploy such large structures. However, their large size, light weight, low flexibility, light damping and nonlinear dynamics creates a unique set of formidable challenges to control system designers. Using the testbed described herein, we hope to validate analytical models, test non-intrusive sensing techniques, and develop control systems for inflatable/rigidizable structures.

\section{REFERENCES}

1.Pappa R.S., Giersch, L.R., Quagliaroli, J.M., “ Photogrammetry of a $5 \mathrm{~m}$ Inflatable Space Antenna with Consumer Digital Cameras." NASA TM 2000-210627, December 2000.

2. Bailey, M.C. Campbell, T.G., Brandt, D., and Cassapakis, C., "Inflatable tensioned membrane waveguide antenna array for space applications." Proceedings of the IUTAN Symposium held in Cambridge, UK, Sept. 6-9, 1998, Pellegrino and Guest Editors.

3.Wilkie, W.K., et al. "Low-Cost Piezocomposite Actuator for Structural Control Applications." $\quad$ SPIE $7^{\text {th }}$ Annual International Symposium on Smart Structures and Materials held in Newport Beach, CA, March 5-9, 2000. 